\title{
OUTER DERIVATIONS OF LIE ALGEBRAS
}

\author{
BY \\ SHIGEAKI TÔGÔ( ${ }^{(1)}$
}

1. Introduction. It is known that certain types of Lie algebras have actually outer derivations. Schenkman and Jacobson [3] have shown that every nonzero nilpotent Lie algebra over a field of arbitrary characteristic has an outer derivation. Leger [4] has shown that if a Lie algebra with nonzero center, over a field of characteristic 0 , has no outer derivations, it is not solvable and its radical is nilpotent. Recently it has been shown by Satô [5] that every nonzero nilpotent Lie algebra over a field of characteristic 0 admits an outer derivation in the radical of its derivation algebra. The purpose of this paper is to generalize and sharpen these results and make a more detailed study of outer derivations of Lie algebras.

We shall consider the class $\mathscr{D}$ of all the Lie algebras $L$ over a field of arbitrary characteristic such that $L \neq[L, L]$ and the center $Z(L) \neq(0)$. All nonzero nilpotent Lie algebras belong to $\mathfrak{D}$. In $\S 3$, we shall show that, if $L \in \mathfrak{D}$ is not the direct sum of the 1-dimensional ideal and an ideal $L_{1}$ such that $L_{1}=\left[L_{1}, L_{1}\right]$ and $Z\left(L_{1}\right)=(0)$, it has a nilpotent outer derivation $D$ with $D^{2}=0$, and otherwise it has a semisimple outer derivation. In order to generalize the above result of Satô, we introduce the notion of Lie algebras of type $(T)$, which will be defined as Lie algebras in $D$ satisfying some additional conditions. $\S 4$ will be devoted to the study of this special type of Lie algebras. In $\S 5$ we shall show that, if $L \in \mathfrak{D}$ is not of type $(T)$, it admits an outer derivation in an abelian ideal of its derivation algebra $\mathscr{D}(L)$, and if $L \in \mathfrak{D}$ is of type $(T)$ and $L^{(1)} \neq L^{(2)}$, it admits a semisimple outer derivation in the radical of $\mathfrak{D}(L)$. Any solvable Lie algebra $L$ of type $(T)$ satisfies the condition $L^{(1)} \neq L^{(2)}$. Hence every solvable Lie algebra with nonzero center admits an outer derivation in the radical of its derivation algebra. In $\$ 6$ we shall show that besides the class $\mathfrak{D}$ there is another class of nonsolvable Lie algebras which have semisimple outer derivations.

2. Preliminaries and notations. Throughout the paper we denote by $\Phi$ a field of arbitrary characteristic unless otherwise stated and by $L$ a finite dimensional Lie algebra over $\Phi$. As usual we put $L^{2}=L^{(1)}=[L, L]$ and $L^{(k)}=\left[L^{(k-1)}, L^{(k-1)}\right]$ for $k \geqq 2$. We denote by $Z(L)$ the center of $L$. The 2-dimensional nonabelian solvable Lie algebra has no outer derivations and its center is (0). Every semisimple Lie algebra $L$ over a field of characteristic 0 has no outer derivations and $L=L^{2}$. Therefore, to investigate the outer derivations of Lie algebras, in this paper we shall mainly consider the following class of Lie algebras.

Received by the editors April 13, 1966.

(1) This work was partially supported by the National Science Foundation. 
$\mathfrak{D}$ : The class of all the Lie algebras $L$ over a field $\Phi$ such that $L \neq L^{2}$ and $Z(L) \neq(0)$.

Every nonzero nilpotent Lie algebra and every solvable Lie algebra with nonzero center belong to this class $\mathfrak{O}$. We denote by ad $x$ the inner derivation associated to $x \in L$. If a subalgebra $H$ of $L$ is stable under ad $x$, then ad $x$ induces the derivation of $H$ which we denote by $\operatorname{ad}_{H} x$. We furthermore employ the following notations.

$\mathfrak{D}(L)$ : The derivation algebra of $L$, that is, the Lie algebra of all the derivations of $L$.

$\Im(L)$ : The ideal of $\mathfrak{D}(L)$ consisting of all the inner derivations of $L$.

$\mathfrak{S}(L)$ : The ideal of $\mathfrak{D}(L)$ consisting of all the central derivations of $L$, that is, the derivations of $L$ which map $L$ into $Z(L)$.

3. Lie algebras in the class $\mathfrak{D}$. We begin with the following

LEMMA 1. Let $L$ be a Lie algebra over a field $\Phi$ such that $Z(L) \neq(0)$ and $M$ be an ideal of $L$ of codimension 1 containing $Z(L)$. Then $[L, Z(M)] \subset Z(M)$ but $[L, Z(M)] \neq Z(M)$.

Proof. Choose an element $e$ of $L$ such that $L=\Phi e+M$. Then $[L, Z(M)]$ $=[e, Z(M)]$. From the fact that $M$ is an ideal of $L$, it follows that $Z(M)$ is stable under ad $e$. Hence $\operatorname{ad}_{Z(M)} e$ is a derivation of $Z(M)$. Since $Z(L) \subset M, Z(L) \subset Z(M)$. Hence the kernel of $\operatorname{ad}_{Z(M)} e$ is $Z(L)$ and therefore is $\neq(0)$. Consequently, $\operatorname{dim} Z(M)$ $>\operatorname{dim}[e, Z(M)]$, from which it follows that $[L, Z(M)] \subset Z(M)$ but $[L, Z(M)]$ $\neq Z(M)$.

THEOREM 1. Every Lie algebra $L \in \mathfrak{D}$ has an outer derivation. More precisely, if $L \in \mathfrak{D}$ is not the direct sum of the 1-dimensional ideal and of an ideal $L_{1}$ such that $L_{1}=L_{1}^{2}$ and $Z\left(L_{1}\right)=(0), L$ has a nilpotent outer derivation $D$ with $D^{2}=0$, and otherwise $L$ has a semisimple outer derivation.

Proof. The case where $L$ is not abelian and has no nonzero abelian direct summands: Since $L \neq L^{2}$, there exists a subspace $M$ of $L$ of codimension 1 containing $L^{2}$. Then $M$ is an ideal of $L$. Since $Z(L) \subset L^{2} \subset M$, it follows from Lemma 1 that $[L, Z(M)] \subset Z(M)$ but $[L, Z(M)] \neq Z(M)$. Choose an element $e$ of $L$ such that $L=\Phi e+M$ and an element $z$ of $Z(M) \backslash[L, Z(M)]$. Define the endomorphism $D$ of $L$ in such a way that $D e=z$ and $D M=(0)$. Then $D$ is a derivation of $L$ such that $D^{2}=0$. Furthermore $D$ is not inner. In fact, suppose that $D$ is inner. Then $D=\operatorname{ad}(\lambda e+x)$ with $\lambda \in \Phi$ and $x \in M$. It follows that $0=D x=[\lambda e+x, x]=\lambda[e, x]$. If $\lambda \neq 0,[e, x]=0$ and therefore $z=D e=[\lambda e+x, e]=0$, contradicting the choice of $z$. Therefore $\lambda=0$. Hence $D=\operatorname{ad} x$ and therefore $x$ is in $Z(M)$. It follows that $z=D e$ $=[x, e] \in[L, Z(M)]$. This again contradicts the choice of $z$. Thus $D$ is not inner.

The case where $L$ is not abelian and has a nonzero abelian direct summand: In this case, $Z(L) \notin L^{2}$. Take a subspace $L_{0}$ of $Z(L)$ complementary to $Z(L) \cap L^{2}$, and choose a subspace $L_{1}$ of $L$ complementary to $L_{0}$ and containing $L^{2}$. Then $L$ is the 
direct sum of the nonzero ideals $L_{0}$ and $L_{1}, L_{0}$ is central and $L_{1}$ has no nonzero abelian direct summands. If $\operatorname{dim} L_{0}>1$, take an arbitrary nonzero endomorphism $D_{0}$ of $L_{0}$ and let $D$ be the trivial extension to $L$. Then $D$ is an outer derivation of $L$. Especially by taking $D_{0}$ in such a way that $D_{0}^{2}=0$, we have a $D$ such that $D^{2}=0$. Now assume that $\operatorname{dim} L_{0}=1$. In the case where $L_{1} \neq L_{1}^{2}$, an arbitrary nonzero endomorphism $D$ of $L$ satisfying the conditions $D L_{1} \subset L_{0}$ and $D\left(L_{0}+L_{1}^{2}\right)=(0)$ is an outer derivation of $L$ such that $D^{2}=0$. In the case where $Z\left(L_{1}\right) \neq(0)$, an arbitrary nonzero endomorphism $D$ of $L$ satisfying the conditions $D L_{0} \subset Z\left(L_{1}\right)$ and $D L_{1}=(0)$ is an outer derivation of $L$ such that $D^{2}=0$. Finally in the case where $L_{1}=L_{1}^{2}$ and $Z\left(L_{1}\right)=(0)$, take a nonzero endomorphism $D_{0}$ of $L_{0}$ and let $D$ be the trivial extension to $L$. Then $D$ is a semisimple outer derivation of $L$.

The case where $L$ is abelian: Every nonzero endomorphism $D$ of $L$ is an outer derivation. If $\operatorname{dim} L>1$, there is a $D$ such that $D^{2}=0$. If $\operatorname{dim} L=1$, every $D$ is semisimple.

COROLlaRY 1. Every solvable Lie algebra whose center is $\neq(0)$ and every nilpotent Lie algebra, of dimension $>1$ over a field $\Phi$, have nilpotent outer derivations $D$ such that $D^{2}=0$.

Proof. A solvable Lie algebra has no nonzero direct summands $L_{1}$ such that $L_{1}=L_{1}^{2}$. Hence the statement follows from Theorem 1 .

As another consequence of Theorem 1, we have the following result which generalizes and sharpens a result of Leger [4, p. 642].

COROLlARY 2. Let $L$ be a Lie algebra over a field of characteristic 0 such that $Z(L) \neq(0)$. Let $R$ be the radical of $L$. Then:

(1) If $L$ has no outer derivations $D$ such that $D^{2}=0$, either $L$ is 1-dimensional, or $L$ is not solvable and $R$ is $[L, R]$ or the direct sum of $[L, R]$ and of the center $Z(L)$ which is 1-dimensional. And then $R$ is nilpotent.

(2) If $L$ has no outer derivations, $L$ is not solvable and $R=[L, R]$.

Proof. Since the basic field of $L$ is of characteristic 0, we take a Levi decomposition $L=S+R$ of $L$. Then $L^{2}=S+[L, R]$ and therefore $[L, R]$ is the radical of $L^{2}$. Furthermore by using Lie's theorem we see that $[L, R]$ is nilpotent. With these in mind we prove

(1) Assume that $L$ has no outer derivations $D$ such that $D^{2}=0$. If $L$ is not 1-dimensional, by Theorem 1 either $L=L^{2}$ or $L$ is the direct sum of a 1-dimensional ideal $L_{0}$ and a nonzero ideal $L_{1}$ such that $L_{1}=L_{1}^{2}$ and $Z\left(L_{1}\right)=(0)$. Hence $L$ is not solvable. In the first case, $R=[L, R]$. In the second case, $L_{0}=Z(L)$ and the radical $R_{1}$ of $L_{1}$ is $\left[L_{1}, R_{1}\right]=[L, R]$. Hence $R$ is the direct sum of $Z(L)$ and $[L, R]$. In both cases $R$ is nilpotent.

(2) If $L$ has no outer derivations $D$ such that $D^{2}=0$ and no semisimple outer derivations, by Theorem 1 we see that $L=L^{2}$. Hence $L$ is not solvable and $R=[L, R]$.

It is to be noted that there are some Lie algebras in the class $\mathfrak{D}$ each of whose 
outer derivations is a linear sum of the outer derivations, which are obtained as in the first part of the proof of Theorem 1, and of the inner derivations. The Lie algebras given in [1, (6), p. 126] and [2] are the examples of such Lie algebras. We omit the illustration.

4. Lie algebras of type $(T)$. In this section we shall study certain types of Lie algebras in the class $\mathfrak{D}$ which play a special rôle in the next section. We call a Lie algebra $L$ over a field $\Phi$ to be of type $(T)$ provided $L$ has a nonzero subspace $S$ satisfying the following conditions:

(1) $L=S+L^{2}, S \cap L^{2}=(0)$.

(2) $\left[S, L^{2}\right]=(0)$.

(3) $[S, S]=\Phi z$ with $0 \neq z \in Z(L)$.

(4) The pairing $\theta$ which assigns to $(x, y) \in S \times S$ the coefficient of $z$ in $[x, y]$ is a nondegenerate (alternate) form on $S$.

If a Lie algebra $L$ of type $(T)$ is solvable, then $L^{(1)}=\Phi z+L^{(2)}$ and therefore $L^{(2)}=L^{(3)}=\cdots=(0)$. Hence $L^{2}=Z(L)=\Phi z$. We call this special type of nilpotent Lie algebras to be pseudo-abelian.

For a Lie algebra $L$ of type $(T), Z(L)=Z\left(L^{2}\right)$. In fact, since $\theta$ is nondegenerate, any nonzero element of $S$ does not commute with some element of $S$. It follows that $Z(L) \subset L^{2}$ and therefore $Z(L) \subset Z\left(L^{2}\right)$. The converse inclusion is evident.

LEMMA 2. Let $L$ be a Lie algebra in the class $\mathfrak{D}$ such that $Z(L) \subset L^{2}$ and $Z(M) \nsubseteq L^{2}$ for every ideal $M$ of codimension 1. Then for every ideal $M$ of codimension 1 there exist an ideal $M^{\prime}$ of codimension 1 and the elements $e$ and $e^{\prime}$ of $L$ satisfying the following conditions:

(1) $L=\Phi e+M=\Phi e^{\prime}+M^{\prime}, e \in Z\left(M^{\prime}\right)\left|L^{2}, e^{\prime} \in Z(M)\right| L^{2}$;

(2) $\left[e, e^{\prime}\right] \neq 0,[e, L]=\left[e^{\prime}, L\right]=\Phi\left[e, e^{\prime}\right] \subset Z(L)$;

(3) $M$ and $M^{\prime}$ have a common basis except $e^{\prime}$ and $e$.

Proof. Take an element $e^{\prime}$ in $Z(M) \backslash L^{2}$ and choose a basis $x_{1}, x_{2}, \ldots, x_{k}$ of a subspace of $M$ complementary to $\Phi e^{\prime}+L^{2}$. Next take a subspace $M^{\prime}$ of $L$ complementary to $\Phi e^{\prime}$ and containing $x_{1}, x_{2}, \ldots, x_{k}$ and $L^{2}$. Then $M^{\prime}$ is an ideal of $L$ of codimension 1. Sirice $Z\left(M^{\prime}\right) \nsubseteq L^{2}$, choose an element $e$ of $Z\left(M^{\prime}\right) \mid L^{2}$. Then $\left[e, e^{\prime}\right] \neq 0$. In fact, if $\left[e, e^{\prime}\right]=0$, then $\left[e, \Phi e^{\prime}+M^{\prime}\right]=(0)$ and therefore $e \in Z(L) \subset L^{2}$, which contradicts the choice of $e$. Hence, together with the fact that $e^{\prime} \in Z(M)$, we see that $e \notin M$, and therefore $L=\Phi e+M$. It follows that $M^{\prime}=\Phi e+\Phi x_{1}+\cdots$ $+\Phi x_{k}+L^{2}$. Thus (1), the first part of (2) and (3) are satisfied by $M^{\prime}, e$ and $e^{\prime}$. We now use (1) and (3) to infer $[e, L]=[e, M]=\Phi\left[e, e^{\prime}\right]$ and similarly $\left[e^{\prime}, L\right]$ $=\Phi\left[e, e^{\prime}\right]$. By using (1) we have

$$
\begin{aligned}
{\left[\left[e, e^{\prime}\right], L\right] } & \subset\left[\left[e, e^{\prime}\right], \Phi e\right]+\left[\left[e, e^{\prime}\right], M\right] \\
& \subset\left[L^{2}, e\right]+\left[[e, M], e^{\prime}\right]+\left[e,\left[e^{\prime}, M\right]\right] \\
& \subset\left[M^{\prime}, e\right]+\left[M, e^{\prime}\right]+\left[e, M^{\prime}\right]=(0) .
\end{aligned}
$$

Hence $\left[e, e^{\prime}\right] \in Z(L)$, completing the proof. 
By making use of Lemma 2 we shall give the following characterization of Lie algebras of type $(T)$.

THEOREM 2. A Lie algebra $L$ over a field $\Phi$ is of type $(T)$ if and only if $L \neq L^{2}$, $(0) \neq Z(L) \subset L^{2}$ and $Z(M) \nsubseteq L^{2}$ for every ideal $M$ of $L$ of codimension 1 .

Proof. Assume that $L$ is of type $(T)$ and let $S, \theta$ be as in the definition. Then $L \neq L^{2}$ and $(0) \neq Z(L) \subset L^{2}$. Every ideal $M$ of $L$ of codimension 1 contains $L^{2}$ and $M \cap S$ is of codimension 1 in $S$. Hence the restriction of $\theta$ to $M \cap S$ has a radical $x \neq 0$ in $M \cap S$. It follows that $x \in S \cap Z(M)$. Therefore $Z(M) \notin L^{2}$.

Conversely, assume that $L \neq L^{2},(0) \neq Z(L) \subset L^{2}$ and $Z(M) \notin L^{2}$ for every ideal $M$ of $L$ of codimension 1 . Let $M$ be a subspace of $L$ of codimension 1 containing $L^{2}$. Then $M$ is an ideal of $L$. Choose an ideal $M^{\prime}$ of codimension 1 and the elements $e$ and $e^{\prime}$ of $L$ as in Lemma 2 . Then we can write $M=\Phi e^{\prime}+\Phi x_{1}+\cdots+\Phi x_{k}+L^{2}$ and $M^{\prime}=\Phi e+\Phi x_{1}+\cdots+\Phi x_{k}+L^{2}$. If $k \geqq 1$, put $M_{1}=\Phi e+\Phi e^{\prime}+\Phi x_{2}+\cdots+\Phi x_{k}+L^{2}$. Then $M_{1}$ is an ideal of $L$ of codimension 1 and $Z\left(M_{1}\right) \subset \Phi x_{2}+\cdots+\Phi x_{k}+L^{2}$ since $\left[e, e^{\prime}\right] \neq 0$. By our assumption $Z\left(M_{1}\right) \notin L^{2}$ and therefore $k \geqq 2$. Now, by using Lemma 2, we choose an ideal $M_{1}^{\prime}$ and the elements $f$ and $f^{\prime}$ of $L$ such that

(1) $L=\Phi f+M_{1}=\Phi f^{\prime}+M_{1}^{\prime}, f \in Z\left(M_{1}^{\prime}\right)\left|L^{2}, f^{\prime} \in Z\left(M_{1}\right)\right| L^{2}$;

(2) $\left[f, f^{\prime}\right] \neq 0,[f, L]=\left[f^{\prime}, L\right]=\Phi\left[f, f^{\prime}\right] \subset Z(L)$;

(3) $M_{1}=\Phi f^{\prime}+\Phi e+\Phi e^{\prime}+\Phi y_{1}+\cdots+\Phi y_{k-2}+L^{2}$ and $M_{1}=\Phi f+\Phi e+\Phi e^{\prime}+\Phi y_{1}+\cdots+\Phi y_{k-2}+L^{2}$.

It is obvious that $f \neq e, f \neq e^{\prime}, f^{\prime} \neq e, f^{\prime} \neq e^{\prime},[f, e]=\left[f, e^{\prime}\right]=\left[f^{\prime}, e\right]=\left[f^{\prime}, e^{\prime}\right]=0$.

Continuing this procedure and changing the notations, we find that

$$
\begin{gathered}
L=\Phi e_{1}+\Phi e_{1^{\prime}}+\Phi e_{2}+\Phi e_{2^{\prime}}+\cdots+\Phi e_{n}+\Phi e_{n^{\prime}}+L^{2}, \\
0 \neq\left[e_{i}, e_{i^{\prime}}\right] \in Z(L),\left[e_{i}, e_{j}\right]=\left[e_{i}, e_{j^{\prime}}\right]=\left[e_{i^{\prime}}, e_{j^{\prime}}\right]=0, \\
{\left[e_{i}, L^{2}\right]=\left[e_{i^{\prime}}, L^{2}\right]=(0) \text { for } i, j=1,2, \ldots, n \text { and } i \neq j .}
\end{gathered}
$$

We now put $z=\left[e_{1}, e_{1^{\prime}}\right]$ and assert that $\left[e_{i}, e_{i^{\prime}}\right]=\alpha_{i} z, \alpha_{i} \neq 0(i=2,3, \ldots, n)$. In fact, put $\left[e_{i}, e_{i^{\prime}}\right]=z_{i}$ and assume that $z$ and $z_{i}$ are linearly independent over $\Phi$. Put

$$
M=\Phi\left(e_{1}+e_{i}\right)+\Phi e_{1^{\prime}}+\Phi e_{i^{\prime}}+\sum_{j \neq 1, i}\left(\Phi e_{j}+\Phi e_{j^{\prime}}\right)+L^{2}
$$

Then $M$ is an ideal of $L$ of codimension 1. Let $x$ be an arbitrary element of $Z(M)$. Since $x$ commutes with $e_{1^{\prime}}, e_{j}, e_{j^{\prime}}(j \neq 1, i), x$ is expressed in the form $x=\lambda e_{1^{\prime}}$ $+\mu e_{i^{\prime}}+y$ with $y \in L^{2}$. It follows that $\left[x, e_{1}+e_{i}\right]=-\lambda z-\mu z_{i}=0$ and therefore $\lambda=\mu=0$. Consequently $x=y \in L^{2}$. Thus $Z(M) \subset L^{2}$, which contradicts our assumption. Therefore $z_{i}=\alpha_{i} z, \alpha_{i} \neq 0$, as was asserted. We now set $S=\Phi e_{1}+\Phi e_{1}+\cdots$ $+\Phi e_{n}+\Phi e_{n^{\prime}}$ and define a bilinear form $\theta$ on $S$ by $[x, y]=\theta(x, y) z$. Then the matrix of $\theta$ with respect to the basis $e_{1}, e_{1^{\prime}}, \ldots, e_{n}, e_{n^{\prime}}$ has the determinant $\alpha_{2}^{2} \alpha_{3}^{2} \cdots \alpha_{n}^{2} \neq 0$. Hence $\theta$ is nondegenerate. The other conditions are obviously satisfied and $L$ is of type $(T)$. Thus the proof is complete. 
Proposition 1. Let $L$ be a Lie algebra of type $(T)$ over a field $\Phi$. Then

$$
Z(\mathfrak{D}(L))=(0) \text {. }
$$

Proof. Suppose $D \in Z(\mathscr{D}(L))$. Since $L$ is of type $(T)$, take $S, z$ and $\theta$ as in the definition. Let $x$ be any nonzero element of $S$. Since $\theta$ is nondegenerate, there is a nonzero element $y$ of $S$ such that $\theta(x, y) \neq 0$ and the set $S_{1}$ of all $u \in S$ such that $\theta(x, u)=0$ is a subspace of $S$ of codimension 1. Put $M=S_{1}+L^{2}$. Then $L=\Phi y+M$ and $x \in Z(M)$. An endomorphism $D^{\prime}$ sending $y$ to $x$ and $M$ into (0) is a derivation of $L$. From the facts that $D \in \mathbb{E}(L)$ and $Z(L) \subset M$, it follows that $D x=\left[D, D^{\prime}\right] y=0$. Therefore $D=0$.

LEMMA 3. Let L be a Lie algebra over a field $\Phi$. Under the same hypotheses as in Lemma $2, \operatorname{dim}[L, Z(M)]=1$ and $\operatorname{dim} Z(M)=\operatorname{dim} Z(L)+1$ for every ideal $M$ of $L$ of codimension 1 .

Proof. Let $M$ be an ideal of $L$ of codimension 1. Choose the elements $e$ and $e^{\prime}$ as in Lemma 2. Then, using Lemma 2, we have

$$
\Phi\left[e, e^{\prime}\right] \subset[L, Z(M)]=[e, Z(M)] \subset[e, L]=\Phi\left[e, e^{\prime}\right]
$$

and therefore $[L, Z(M)]=[e, Z(M)]=\Phi\left[e, e^{\prime}\right] \neq(0)$. Hence $\operatorname{dim}[L, Z(M)]=1$. Thus the rank of $\operatorname{ad}_{z(M)} e$ is 1 . But the kernel of $\operatorname{ad}_{z(M)} e$ is $Z(L)$. Hence

$$
\operatorname{dim} Z(M)=\operatorname{dim} Z(L)+1 \text {. }
$$

Proposition 2. A Lie algebra $L$ over a field $\Phi$ is one of type $(T)$ such that $\operatorname{dim} Z(L)=1$ if and only if $L \neq L^{2},(0) \neq Z(L) \subset L^{2}$ and $[L, Z(M)]=Z(M) \cap L^{2}$ for every ideal $M$ of $L$ of codimension 1.

Proof. Assume that $L$ is a Lie algebra of type $(T)$ such that $\operatorname{dim} Z(L)=1$. Then $L \neq L^{2}$ and $(0) \neq Z(L) \subset L^{2}$. For every ideal $M$ of codimension $1, Z(M)$ is a characteristic ideal of $M$ and therefore $[L, Z(M)] \subset Z(M) \cap L^{2} \subset Z\left(L^{2}\right)=Z(L)$. Since $\operatorname{dim}[L, Z(M)]=1$ by Theorem 2 and Lemma 3 and since $\operatorname{dim} Z(L)=1$ by assumption, it follows that $[L, Z(M)]=Z(M) \cap L^{2}$.

Conversely, assume that $L \neq L^{2},(0) \neq Z(L) \subset L^{2}$ and $[L, Z(M)]=Z(M) \cap L^{2}$ for every ideal $M$ of codimension 1. If $Z(M) \subset L^{2}$ for some ideal $M$ of codimension 1, $[L, Z(M)]=Z(M)$ and this contradicts Lemma 1 since $Z(L) \subset L^{2} \subset M$. Hence $Z(M) \nsubseteq L^{2}$ for every ideal $M$ of $L$ of codimension 1. By Theorem 2, $L$ is of type $(T)$. Now we have $Z(L)=Z\left(L^{2}\right) \subset Z(M) \cap L^{2}=[L, Z(M)]$. Since $Z(L) \neq(0)$ and since $\operatorname{dim}[L, Z(M)]=1$ by Lemma 3, it follows that $\operatorname{dim} Z(L)=1$.

Proposition 3. Let $L$ be a Lie algebra over a field $\Phi$. Then each of the following conditions is necessary and sufficient for $L$ to be pseudo-abelian:

(1) $Z(L)=L^{2}$ and $\operatorname{dim} Z(L)=1$.

(2) $Z(L)=L^{2}$ and $\operatorname{dim} Z(M)=2 \operatorname{dim} Z(L)$ for every ideal $M$ of $L$ of codimension 1 .

(3) $Z(L)=L^{2}$ and $\operatorname{dim}\left(Z(M) \cap L^{2}\right)=1$ for every ideal $M$ of $L$ of codimension 1 . 
Proof. Assume that $L$ satisfies the conditions in (1). Let $S$ be any subspace of $L$ complementary to $L^{2}$. Then the bilinear form $\theta$ on $S$ defined by $[x, y]=\theta(x, y) z$ with $0 \neq z \in Z(L)$ is nondegenerate since $Z(L)=L^{2}$. S obviously satisfies the other conditions so that $L$ is pseudo-abelian. The converse is evident.

If $L$ is pseudo-abelian, by Lemma 3 we see that $\operatorname{dim} Z(M)=\operatorname{dim} Z(L)+1$ for every ideal $M$ of codimension 1 . Since $\operatorname{dim} Z(L)=1$, it follows that $\operatorname{dim} Z(M)$ $=2 \operatorname{dim} Z(L)$. Conversely, assume that $L$ satisfies the conditions in (2). Then $Z(M) \notin L^{2}$ for every ideal $M$ of $L$ of codimension 1. By Theorem 2, $L$ is of type $(T)$. But $L$ is solvable since $Z(L)=L^{2}$. Thus $L$ is pseudo-abelian.

If $Z(L)=L^{2}, L$ is nilpotent. Every ideal $M$ of $L$ of codimension 1 contains $L^{2}$ and therefore $Z(L) \subset Z(M)$. It follows that $Z(L)=Z(M) \cap L^{2}$. Hence the condition (3) is equivalent to the condition (1).

As an immediate consequence of Proposition 3 we have the following

Corollary. Let $L$ be a pseudo-abelian Lie algebra over a field $\Phi$. Then for every ideal $M$ of codimension $1, \operatorname{dim} Z(M)=2$ and $Z(L)=Z(M) \cap L^{2}$.

Here we give an example of nonsolvable Lie algebras of type $(T)$. Let $L$ be the Lie algebra over a field of characteristic $\neq 2$ described in terms of a basis $e_{1}, e_{2}, \ldots$, $e_{8}$ by the multiplication table:

$$
\begin{array}{lll}
{\left[e_{1}, e_{2}\right]=2 e_{2},} & {\left[e_{1}, e_{3}\right]=-2 e_{3},} & {\left[e_{2}, e_{3}\right]=e_{1},} \\
{\left[e_{1}, e_{6}\right]=-e_{6},} & {\left[e_{1}, e_{7}\right]=e_{7},} & {\left[e_{2}, e_{6}\right]=-e_{7},} \\
{\left[e_{3}, e_{7}\right]=-e_{6},} & {\left[e_{4}, e_{5}\right]=e_{8},} & {\left[e_{6}, e_{7}\right]=e_{8} .}
\end{array}
$$

In addition $\left[e_{i}, e_{j}\right]=-\left[e_{j}, e_{i}\right]$ and, for $i<j,\left[e_{i}, e_{j}\right]=0$ if it is not in the table above. Then $L$ is obviously such a Lie algebra.

In connection with the statements of Proposition 3 and its corollary, one might expect that $L$ is pseudo-abelian if $Z(L)=L^{2}=Z(M) \cap L^{2}$ and $\operatorname{dim} Z(M)=2$ for every ideal $M$ of $L$ of codimension 1 . However this does not hold in general. We shall show this fact by the following example.

Let $L$ be the Lie algebra over the field of real numbers described in terms of a basis $e_{1}, e_{2}, \ldots, e_{6}$ by the following multiplication table:

$$
\begin{aligned}
& {\left[e_{1}, e_{2}\right]=e_{5}, \quad\left[e_{1}, e_{3}\right]=e_{6}, \quad\left[e_{1}, e_{4}\right]=e_{6},} \\
& {\left[e_{3}, e_{4}\right]=e_{5}, \quad\left[e_{2}, e_{3}\right]=e_{6} .}
\end{aligned}
$$

In addition $\left[e_{i}, e_{j}\right]=-\left[e_{j}, e_{i}\right]$ and, for $i<j,\left[e_{i}, e_{j}\right]=0$ if it is not in the table above. For any ideal $M$ of $L$ of codimension 1, we have $Z(M)=Z(L)$. The original proof of this fact contained a long computation and the following short proof was suggested by the referee. Suppose that $f \in Z(M)$. Then $f=\sum_{i=1}^{6} \alpha_{i} e_{i}$. Since ad $f$ has rank $\leqq 1,\left[f, e_{2}\right]$ and $\left[f, e_{4}\right]$ are linearly dependent, from which it follows that $\alpha_{1}^{2}+\alpha_{3}^{2}=0$ and therefore $\alpha_{1}=\alpha_{3}=0$. Similarly from the linear dependence of 
$\left[f, e_{1}\right]$ and $\left[f, e_{3}\right]$ we have $\alpha_{2}=\alpha_{4}=0$. Hence $f \in Z(L)$ and $Z(M)=Z(L)$. We see that $L$ satisfies the conditions stated above, but $L$ is not pseudo-abelian.

5. Ideals of the derivation algebras containing outer derivations. In this section, by making use of Theorem 2 in $\S 4$ we shall show some results generalizing and sharpening a result of Satô [5].

Through this section, we denote by $\mathfrak{R}_{0}$ (resp. $\mathfrak{S}_{0}$ ) the set of all the derivations of $L$ which map $L$ into $L^{2}$ (resp. $Z(L)$ ) and $L^{2}$ (resp. $Z(L)$ ) into (0). Then from the fact that $L^{2}$ and $Z(L)$ are characteristic ideals of $L$, it follows that $\mathfrak{R}_{0}$ and $\mathfrak{\mho}_{0}$ are abelian ideals of $\mathfrak{D}(L)$.

THEOREM 3. Let $L$ be a nonabelian Lie algebra in the class $\mathfrak{D}$.

(1) If $L$ has no nonzero abelian direct summands and if $L$ is not of type $(T)$, then $L$ has an outer derivation in $\mathfrak{R}_{0}$.

(2) Assume that $L$ has a nonzero abelian direct summand.

(a) If $Z(L)$ is not a direct summand of $L$, then $L$ has an outer derivation in $\mathfrak{N}_{0} \cap \mathfrak{S}(L)$.

(b) If $Z(L)$ is a direct summand of $L$ and $L / Z(L)$ does not coincide with the derived algebra, then $L$ has an outer derivation in $\mathfrak{夭}_{0}$.

(c) If $Z(L)$ is a direct summand of $L$ and $L / Z(L)$ coincides with the derived algebra, then $L$ has an outer derivation in $Z(\mathfrak{D}(L))$.

(3) If $L$ is of type $(T)$ and $L^{(1)} \neq L^{(2)}$, then $L$ has an outer derivation in the radical of $\mathfrak{D}(L)$.

Proof. (1) Assume that $L$ has no nonzero abelian direct summands and $L$ is not of type $(T)$. Then $Z(L) \subset L^{2}$. Therefore by Theorem 2 there exists an ideal $M$ of $L$ of codimension 1 such that $Z(M) \subset L^{2}$. Every such $M$ contains $L^{2}$ and therefore $Z(L)$. By Lemma $1[L, Z(M)] \subset Z(M)$ but $[L, Z(M)] \neq Z(M)$. As in the first part of the proof of Theorem 1 we can show that every endomorphism $D$ of $L$ defined in such a way that $D L \subset Z(M) \backslash[L, Z(M)]$ and $D M=(0)$ is an outer derivation of $L$, which belongs to $\Re_{0}$ since $Z(M) \subset L^{2} \subset M$.

(2) Assume that $L$ has a nonzero abelian direct summand. Then, as in the second part of the proof of Theorem 1 , we see that $L$ is the direct sum of a nonzero abelian ideal $L_{1}$ and a nonzero ideal $L_{2}$ such that $Z\left(L_{2}\right) \subset L_{2}^{2}$. If $Z(L)$ is not a direct summand of $L$, then $Z\left(L_{2}\right) \neq(0)$. Every nonzero endomorphism $D$ of $L$ defined in such a way that $D L_{1} \subset Z\left(L_{2}\right)$ and $D L_{2}=(0)$ is an outer derivation of $L$ belonging to $\Re_{0} \cap \mathbb{E}(L)$. If $Z(L)$ is a direct summand and $L / Z(L)$ does not coincide with its derived algebra, then $Z(L)=L_{1}$ and $L_{2} \neq L_{2}^{2}$. Every nonzero endomorphism $D$ of $L$ such that $D L_{2} \subset L_{1}$ and $D\left(L_{1}+L_{2}^{2}\right)=(0)$ is an outer derivation of $L$ belonging to $\mho_{0}$. If $Z(L)$ is a direct summand and $L / Z(L)$ coincides with its derived algebra, then $Z(L)=L_{1}$ and $L_{2}=L_{2}^{2}$. The trivial extension of the identity endomorphism of $L_{1}$ to $L$ is an outer derivation belonging to $Z(\mathfrak{D}(L))$.

(3) Assume that $L$ is a Lie algebra of type $(T)$ such that $L^{(1)} \neq L^{(2)}$. Let $S$ and $z$ be as in the definition of Lie algebras of type $(T)$. Then $L^{(1)}=\Phi z+L^{(2)}$ and $z \notin L^{(2)}$. 
Put $L_{1}=S+\Phi z$ and $L_{2}=L^{(2)}$. Then $L_{1}$ is pseudo-abelian and $L$ is the direct sum of the ideals $L_{1}$ and $L_{2}$. In the case where $L_{2} \neq(0)$, define an endomorphism $D_{0}$ of $L$ in such a way that

$$
\begin{aligned}
D_{0} x & =x \text { for } x \in S, \\
D_{0} z & = \begin{cases}2 z & \text { if the characteristic of } \Phi \text { is } \neq 2, \\
0 & \text { if the characteristic of } \Phi \text { is } 2,\end{cases} \\
D_{0} L_{2} & =(0) .
\end{aligned}
$$

Then $D_{0}$ is a semisimple outer derivation of $L$. Putting $\Re_{0}=\Phi D_{0}+\Subset(L)$, we assert that $\Re_{0}$ is a solvable ideal of $\mathfrak{D}(L)$. In fact, since $Z(L) \subset L^{2}, \mathfrak{S}(L)$ is an abelian ideal of $\mathscr{D}(L)$. Hence $\Re_{0}$ is a solvable subalgebra of $\mathscr{D}(L)$. Let $D$ be an arbitrary derivation of $L$. For any $x \in S$, write $D x=y+\lambda z+u$ with $y \in S$ and $u \in L_{2}$. Then $u \in Z\left(L_{2}\right)$ $\subset Z(L)$. Therefore, if the characteristic of $\Phi$ is $\neq 2$ (resp. is 2 ), we have

$$
\left[D, D_{0}\right] x=D x-D_{0} D x=-\lambda z+u
$$

(resp. $\lambda z+u$ ). Hence $\left[D, D_{0}\right] x \in Z(L)$. Since $Z(L)$ and $L^{(2)}$ are characteristic ideals of $L$, we have $\left[D, D_{0}\right] z \in Z(L)$ and $\left[D, D_{0}\right] L_{2} \subset D_{0} D L_{2} \subset D_{0} L_{2}=(0)$. Therefore we conclude that $\left[D, D_{0}\right] \in \mathfrak{C}(L)$. Thus $\Re_{0}$ is a solvable ideal of $\mathfrak{D}(L)$, as was asserted. In the case where $L_{2}=(0)$, define $D_{0}$ and $\Re_{0}$ similarly. Then $D_{0}$ is a semisimple outer derivation of $L$ and $\Re_{0}$ is a solvable ideal of $\mathscr{D}(L)$. Thus in both cases we see that there is an outer semisimple derivation in the radical of $\mathfrak{D}(L)$. The proof is complete.

Here we shall show that there is an example of Lie algebras $L$ satisfying the hypotheses in each case of Theorem 3 such that the indicated ideal of $\mathfrak{D}(L)$ contains no ideals of $\mathfrak{D}(L)$ different from itself and containing outer derivations.

Let $L$ be the Lie algebra over a field $\Phi$ of characteristic $\neq 2$ described in terms of a basis $e_{1}, e_{2}, e_{3}, e_{4}$ by the multiplication table

$$
\begin{aligned}
& {\left[e_{1}, e_{2}\right]=e_{2}, \quad\left[e_{1}, e_{3}\right]=-e_{3}, \quad\left[e_{2}, e_{3}\right]=e_{4},} \\
& {\left[e_{i}, e_{4}\right]=0 \text { for } i=1,2,3 .}
\end{aligned}
$$

Then $L$ satisfies the hypotheses in the case (1) of Theorem 3. Let $D$ be any derivation of $L$ and put $D e_{i}=\sum_{j=1}^{4} \lambda_{i j} e_{j}(i=1,2,3,4)$. Then the matrix of $D$ is

$$
\left(\begin{array}{cccc}
0 & \lambda_{12} & \lambda_{13} & \lambda_{14} \\
0 & \lambda_{22} & 0 & -\lambda_{13} \\
0 & 0 & \lambda_{33} & -\lambda_{12} \\
0 & 0 & 0 & \lambda_{22}+\lambda_{33}
\end{array}\right) .
$$

Denote by $D_{i j}$ the derivation of $L$ whose matrix is obtained from the above matrix by putting $\lambda_{i j}=1$ and $\lambda_{k l}=0$ for all $(k, l) \neq(i, j)$. Then $\mathfrak{N}_{0}=\Phi D_{14}$ where $D_{14}$ is outer. 
Let $L$ be the Lie algebra over a field $\Phi$ described in terms of a basis $e_{1}, e_{2}, e_{3}, e_{4}$ by the multiplication table

$$
\begin{aligned}
& {\left[e_{1}, e_{i}\right]=0 \text { for } i=2,3,4,} \\
& {\left[e_{2}, e_{3}\right]=e_{4}, \quad\left[e_{2}, e_{4}\right]=\left[e_{3}, e_{4}\right]=0 .}
\end{aligned}
$$

Then $L$ satisfies the hypotheses in the case (2)(a) of Theorem 3. Since $L^{2} \subset Z(L)$, $\mathfrak{R}_{0} \subset \mathfrak{C}(L)$ and therefore $\mathfrak{R}_{0} \cap \mathfrak{E}(L)=\mathfrak{N}_{0}$. Let $D$ be any derivation of $L$. Using the similar notations $\lambda_{i j}$ and $D_{i j}$ as above, the matrix of $D$ is

$$
\left(\begin{array}{cccc}
\lambda_{11} & 0 & 0 & \lambda_{14} \\
\lambda_{21} & \lambda_{22} & \lambda_{23} & \lambda_{24} \\
\lambda_{31} & \lambda_{32} & \lambda_{33} & \lambda_{34} \\
0 & 0 & 0 & \lambda_{22}+\lambda_{33}
\end{array}\right) .
$$

Then $\mathfrak{R}_{0}=\Phi D_{14}+\Phi D_{24}+\Phi D_{34}$, where $D_{14}$ is outer and $D_{24}, D_{34}$ are inner. Let $\mathfrak{M}$ be an ideal of $\mathfrak{D}(L)$ which is contained in $\mathfrak{N}_{0}$ and contains outer derivations. Then $\mathfrak{M}$ contains $D_{14}+\lambda D_{24}+\mu D_{34}$. Since $\left[D_{14}+\lambda D_{24}+\mu D_{34}, D_{21}\right]=D_{24}$ and $\left[D_{24}, D_{32}\right]=D_{34}, \mathfrak{M}$ coincides with $\mathfrak{R}_{0}$.

Let $L$ be the direct sum of the 1-dimensional Lie algebra and the 2-dimensional nonabelian solvable Lie algebra over a field $\Phi$. Then $L$ satisfies the hypotheses in the case (2)(b) of Theorem 3 and $\mathfrak{S}_{0}$ is 1-dimensional.

Let $L$ be the direct sum of the 1-dimensional Lie algebra and a semisimple Lie algebra over a field of characteristic 0 . Then $L$ satisfies the hypotheses in the case (2)(c) of Theorem 3 and $Z(\mathfrak{D}(L))$ is 1-dimensional.

Finally, let $L$ be a pseudo-abelian Lie algebra over a field $\Phi$ of characteristic $\neq 2$. Then $L$ satisfies the hypotheses in the case (3) of Theorem 3. Let $S, z$ and $\theta$ be as in the definition of pseudo-abelian Lie algebras. Since $\theta$ is nondegenerate, $\mathfrak{C}(L)$ $=\Im(L)$. Let $\subseteq$ be the set of all endomorphisms of $S$ which are skew relative to $\theta$. Then $\subseteq$ is a symplectic Lie algebra. Take the set of trivial extensions of elements of $\subseteq$ to $L$, which we again denote by $\subseteq$. Then $\subseteq \subset \mathfrak{D}(L)$. Let $D_{0}$ and $\Re_{0}$ be as in the third part of the proof of Theorem 3. We assert that $\mathfrak{D}(L)=\subseteq \mathfrak{\Re}_{0}$. In fact, any derivation $D$ of $L$ induces the endomorphism $D^{\prime}$ of $L$ mapping $S$ into $Z(L)$ and $Z(L)$ into (0). $D^{\prime}$ is in $\subseteq(L)$. We denote by $\lambda$ the coefficient of $z$ in $D z$ and put $D_{1}=D-(\lambda / 2) D_{0}-D^{\prime}$. Then it is immediate that $D_{1} \in \mathcal{S}$ and we have the assertion. Thus $\Re_{0}$ is the radical of $\mathfrak{D}(L)$. Since $\left[D^{\prime}, D_{0}\right]=D^{\prime}$ for any $D^{\prime}$ in $\Im(L), \Re_{0}$ contains no ideals of $\mathfrak{D}(L)$ different from $\Re_{0}$ and containing outer derivations.

THEOREM 4. Let $L$ be a solvable Lie algebra over a field $\Phi$ such that $Z(L) \neq(0)$.

(1) If $L$ is not abelian and not pseudo-abelian and if $L$ has no nonzero abelian direct summands, then $L$ has an outer derivation in $\mathfrak{N}_{0}$.

(2) Assume that $L$ is not abelian and has a nonzero abelian direct summand. If $Z(L)$ is not a direct summand of $L$, then $L$ has an outer derivation in $\mathfrak{R}_{0} \cap \mathcal{E}(L)$. If $Z(L)$ is a direct summand of $L$, then $L$ has an outer derivation in $\mathfrak{E}_{0}$. 
(3) If $L$ is abelian, $L$ has an outer derivation in $Z(\mathfrak{D}(L))$. If $L$ is pseudo-abelian, $L$ has an outer derivation in the radical of $\mathfrak{D}(L)$.

Proof. Let $L$ be a solvable Lie algebra over $\Phi$ such that $Z(L) \neq(0)$. Then $L \neq L^{2}$. We do not have the case (c) in the statement (2) of Theorem 3. If $L$ is of type $(T)$, then $L$ is pseudo-abelian and $L^{(1)} \neq L^{(2)}$. The statement is evident for the case where $L$ is abelian. Thus the theorem follows from Theorem 3.

Corollary 1. Let L be a Lie algebra in the class $\mathfrak{D}$. If $L$ is not of type $(T), L$ has an outer derivation in an abelian ideal of $\mathfrak{D}(L)$.

Proof. This is immediate from Theorems 3 and 4.

COROLLARY 2. Every Lie algebra $L$ such that $L \neq L^{(1)}, L^{(1)} \neq L^{(2)}$ and $Z(L) \neq(0)$, every solvable Lie algebra $L$ such that $Z(L) \neq(0)$, and every nonzero nilpotent Lie algebra, over a field $\Phi$, have outer derivations in the radicals of their derivation algebras.

Proof. This is immediate from Theorems 3 and 4.

Corollary 3. Let $L$ be a nilpotent Lie algebra over a field $\Phi$. Assume that $L$ is neither abelian nor pseudo-abelian. Then $L$ has an outer derivation in $\mathfrak{N}_{0}$ and especially in $\mathfrak{R}_{0} \cap \mathfrak{E}(L)$ if $L$ has a nonzero direct summand different from itself.

Proof. Owing to Theorem 4 it suffices to prove the statement in the case where $L$ has a nonzero direct summand different from itself. In this case $L$ has a nonabelian direct summand $L_{1}$ which is different from $L$. If $Z\left(L_{1}\right) \nsubseteq L_{1}^{2}$, as in the second part of the proof of Theorem $1, L_{1}$ is the direct sum of a central ideal and a nonzero ideal $L_{0}$ such that $Z\left(L_{0}\right) \subset L_{0}^{2}$. Thus we may assume that $Z\left(L_{1}\right) \subset L_{1}^{2}$. Denote by $L_{2}$ the ideal of $L$ complementary to $L_{1}$. Then $L_{2} \neq(0)$. Define a nonzero endomorphism $D$ of $L$ in such a way that $D L_{1}=(0), D L_{2} \subset Z\left(L_{1}\right)$ and $D L_{2}^{2}=(0)$. Then $D$ is an outer derivation belonging to $\mathfrak{R}_{0} \cap \mathfrak{E}(L)$.

6. Nonsolvable Lie algebras which have semisimple outer derivations. Besides the class $\mathfrak{D}$ of Lie algebras considered in $\S \S 3,4$ and 5 , there is another class of Lie algebras which have outer derivations. For example, let $L_{1}$ be a simple Lie algebra and $M$ be a faithful irreducible $L_{1}$-module. Regarding $M$ as an abelian Lie algebra, we put $L=L_{1}+M$ (semidirect sum). Then the endomorphism of $L$ acting as identity on $M$ and zero on $L_{1}$ is a semisimple derivation of $L$ which is obviously not inner.

In the rest of this section, we assume that $L$ is a Lie algebra over a field of characteristic 0 . For a maximal semisimple subalgebra $S$ of $L$, we denote by $\mathfrak{A}(S)$ the set of all derivations of $L$ which map $S$ into (0). Then we have

Lemma 4. Let $R$ be the radical of $L$ and let $L=S+R$ be a Levi decomposition of $L$. Then among maximal toroidal subalgebras of the radical of $\mathscr{D}(R)$ there exists one which can be imbedded in $\mathfrak{X}(S)$.

Proof. Let $\mathfrak{S}$ be a maximal semisimple subalgebra of $\mathscr{D}(R)$ containing $\operatorname{ad}_{R} S$. Since $\mathscr{D}(R)$ is algebraic, there exists a maximal toroidal subalgebra $\mathfrak{T}$ of the radical 
of $\mathfrak{D}(R)$ such that $[\mathfrak{S}, \mathfrak{T}]=(0)$. Hence $\left[\operatorname{ad}_{R} S, \mathfrak{T}\right]=(0)$. For any $D$ in $\mathfrak{T}$ we have $D[s, r]=[s, D r]$ for any $s \in S$ and any $r \in R$. Therefore $D$ can be extended to be a derivation of $L$ by putting $D s=(0)$. Thus $\mathfrak{I}$ can be imbedded in $\mathfrak{A}(S)$.

THEOREM 5. Let $L$ be a nonsolvable Lie algebra over a field of characteristic 0. Let $R$ be the radical of $L$. Then, if $R$ has a semisimple outer derivation in the radical of $\mathfrak{D}(R), L$ has a semisimple outer derivation. Especially, in the case where $R$ is nilpotent, if $R$ has a semisimple derivation in the radical of $\mathfrak{D}(R)$, then $L$ has a semisimple outer derivation.

Proof. Let $L=S+R$ be a Levi decomposition of $L$. Let $\Re$ be the radical of $\mathscr{D}(R)$. By Lemma 4 there exists a maximal toroidal subalgebra $\mathfrak{T}$ of $\mathfrak{R}$ which can be imbedded in $\mathfrak{A}(S)$. Assume that $D^{\prime}$ is a semisimple outer derivation belonging to $\Re$. Then there exists an element $E$ of $\Re^{2}$ such that $D_{1}^{\prime}=\sigma D^{\prime} \in \mathfrak{I}$ with $\sigma=\exp \left(\operatorname{ad}_{\Re} E\right)$. Let $D_{1}$ be the derivation in $\mathfrak{A}(S)$ which is a trivial extension of $D_{1}^{\prime}$. Then $D_{1}$ is a semisimple derivation of $L$. Furthermore $D_{1}$ is not inner. In fact, if $D_{1}$ is inner, $D_{1}=\operatorname{ad}(s+r)$ with $s \in S$ and $r \in R$. It follows that $D_{1} S=[s+r, S]=(0)$ and therefore $[s, S]=[r, S] \subset S \cap R=(0)$. Hence $s=0$ and $D_{1}=\operatorname{ad} r$. It follows that $D_{1}^{\prime}=\operatorname{ad}_{R} r$ and therefore $D^{\prime}=\sigma^{-1} D_{1}^{\prime}=\operatorname{ad}_{R} u$ with $u=(\exp (-E)) r$, which contradicts our assumption. Thus $D_{1}$ is a semisimple outer derivation of $L$ and the first statement is proved. In the case where $R$ is nilpotent, every semisimple derivation of $R$ is outer. Hence the second statement is immediate from the first one.

COROLLARY. Let $L$ be a Lie algebra over a field of characteristic 0 whose radical $R$ is nilpotent. If $R$ has a derivation whose trace is $\neq 0$, then $L$ has a semisimple outer derivation. In particular, this is the case if $R$ is quasi-cyclic.

Proof. Assume that $L$ has no semisimple outer derivations. In the case where $L$ is not solvable, by Theorem 5 we see that $R$ has no semisimple derivations in the radical $\mathfrak{R}$ of $\mathfrak{D}(R)$. Let $\mathfrak{D}(R)=\subseteq+\Re$ be a Levi decomposition of $\mathfrak{D}(R)$. Then $\Re$ is splittable and therefore consists of nilpotent elements. Since $\subseteq=[\subseteq, \subseteq]$, the trace of every derivation of $R$ is 0 . In the case where $L$ is solvable, $L=R$ and therefore $R$ has no semisimple derivations. Since $\mathfrak{D}(R)$ is splittable, $\mathscr{D}(R)$ consists of only nilpotent derivations. Hence the trace of every derivation of $R$ is 0 . Thus the first statement is proved.

If $R$ is quasi-cyclic, there is a subspace $U$ of $R$ such that $R=\sum_{i} U^{i}$ with $U^{i} \cap U^{j}$ $=(0)$ for $i \neq j$, where $U^{1}=U$ and $U^{i}=\left[U, U^{i-1}\right]$ for $i \geqq 2$. Define the endomorphism $D$ of $R$ in such a way that $D u=i u$ for $u \in U^{i}(i=1,2, \ldots)$. Then $D$ is a derivation of $R$ whose trace is $\neq 0$.

\section{REFERENCES}

1. N. BourbaKI, Groupes et algèbres de Lie, Hermann, Paris, 1960.

2. J. Dixmier and W. G. Lister, Derivations of nilpotent Lie algebras, Proc. Amer. Math. Soc. 8 (1957), 155-158. 
3. N. Jacobson, A note on automorphisms and derivations of Lie algebras, Proc. Amer. Math. Soc. 6. (1955), 281-283.

4. G. Leger, Derivations of Lie algebras. III, Duke Math. J. 30 (1963), 637-645.

5. T. Satô, On derivations of nilpotent Lie algebras, Tôhoku Math. J. 17 (1965), 244-249.

University of California,

Berkeley, California

HiROSHMMA UNIVERSITY,

Hiroshma, JAPAN 